\title{
Ingeniería didáctica y su influencia en el aprendizaje de funciones en los estudiantes de la Universidad Nacional Micaela Bastidas de Apurímac
}

\section{Didactic engineering and his influence on learning of functions in the students of the National University of Apurimac's Micaela Bastidas}

\author{
Alejandro Manuel Ecos Espino \\ Universidad Nacional Micaela Bastidas de Apurímac, Av. Arenas 123, Abancay, Apurímac
}

DOI: https://doi.org/10.33017/RevECIPeru2013.0012/

\begin{abstract}
Resumen
En esta investigación se presentan los resultados de la aplicación de una ingeniería didáctica, diseñada con el objetivo de mejorar el aprendizaje del concepto de función en los estudiantes de la Universidad Nacional Micaela Bastidas de Apurímac. Para el diseño de la ingeniería se tomó como marco de referencia la Teoría de las Situaciones Didácticas propuesta por G. Brosseau y la Teoría de los Registros de Representación Semiótica planteada por R. Duval. Se llevó a cabo un análisis cognitivo sobre las concepciones de los estudiantes en relación al concepto de función; un análisis didáctico sobre la forma como se trataba este concepto en los planes de estudio y en los libros de texto; y un análisis epistemológico para identificar como el concepto fue evolucionando hasta llegar a una definición más elaborada. El análisis anterior permitió la identificación de las variables significativas a evaluar, así como el diseño de 6 situaciones didácticas que fueron trabajadas con los estudiantes. El proceso de experimentación se llevó a cabo con la participación de los estudiantes del primer ciclo de estudios de la Escuela académico Profesional de Ingeniería Agroindustrial, a los cuales se les aplicó inicialmente un pre test; y un post test después de la experimentación. Los resultados obtenidos nos muestran que la aplicación de la ingeniería didáctica permitió mejorar significativamente el aprendizaje de los estudiantes sobre el concepto de función, en relación al manejo de los registros de representación verbal, tabular, gráfica y algebraica, con un 95\% de confianza, validada a través de la prueba estadística T de Students. Además, se verificó que la aplicación de la ingeniería didáctica permitió al $91 \%$ de los estudiantes superar el nivel regular de aprendizaje, así como mejorar las diferentes habilidades que le permiten realizar conversiones entre los distintos registros de representación del concepto de función, tales como: interpretación de gráficas, interpretación de fórmulas, realizar ajustes numéricos, calcular valores, entre otros.
\end{abstract}

Descriptores: Ingeniería Didáctica, Representación, Función.

\section{Abstract}

This research presents the results of the application of a didactic engineering, designed with the aim of improving learning the concept of function in students of the National University of Apurimac Micaela Bastidas. For engineering design took as reference the Theory of Didactic Situations proposed by G. Brosseau and the Theory of Semiotics Representation Records posed by R. Duval. They conducted a cognitive analysis of the conceptions of students regarding the concept of function, a training analysis on how this concept was in the curricula and textbooks, and an epistemological analysis to identify how the concept was to evolve into a more elaborate definition. The above analysis allowed the identification of significant variables and evaluate the design of six teaching situations were worked with students. The experimental process was carried out with the participation of students of lower academic studies at the School of Engineering Professional Agroindustrial, to which they are initially applied a pre-test, and post test after experimentation. The results obtained show that the application of didactic engineering allowed significantly improve student learning about 
the concept of function in relation to the management of records of verbal representation, tabular, graphical and algebraic, with $95 \%$ confidence, validated through the test statistic T Students. Furthermore, it was verified that the application of didactic engineering allowed the $91 \%$ of students regularly exceed the level of learning and improve the various skills that will enable conversion between different registers of representation of the concept of function, such as interpretation graphs, interpreting formulas, numerical adjustments, calculate values, among others.

\section{Keywords: Teaching Engineering, Representation, Function}

\section{Introducción}

El concepto de función es una herramienta muy importante para modelar diferentes situaciones de la vida real. Su aprendizaje adecuado permitirá al estudiante adquirir esta habilidad, así como desempeñarse sin muchas dificultades en asignaturas de ciclos superiores propias del pensamiento matemático avanzado.

Los estudiantes de la Universidad Nacional Micaela Bastidas de Apurímac manifiestan serias dificultades en cuanto a su aprendizaje. En relación a la asignatura de Matemática Básica, se supone que los estudiantes ingresan a la Universidad con todas las capacidades matemáticas necesarias para acceder a las complejas estructuras que se estudian en la Universidad.

EI DCN 2009 con el cual se desarrolla la educación obligatoria sostiene que: "Ser competente matemáticamente supone tener habilidad para usar los conocimientos con flexibilidad y aplicar con propiedad lo aprendido en diferentes contextos". En cuanto al concepto de función, el DCN 2009 plantea una temática bastante similar a la que se trabaja en la Universidad, postulando que el estudiante al terminar su $5^{\circ}$ grado: "Resuelve problemas de programación lineal y funciones; argumenta y comunica los procesos de solución y resultados utilizando lenguaje matemático", situación que gran parte de los estudiantes no refleja en el trabajo dentro de las aulas universitarias, lo cual pone en tela de juicio la efectividad de la enseñanza recibida por nuestros estudiantes.

Por otro lado, dentro de la Universidad, la enseñanza de cursos de los matemática ha estado fuertemente influenciada por la Matemática Moderna y el estilo Bourbakista en la instrucción matemática; situación que, lejos de mejorar el aprendizaje de esta importante materia, ha colaborado en el fortalecimiento de concepciones negativas de muchos estudiantes hacia esta ciencia, impregnando la idea que la matemática es una ciencia en la cual sólo se deben aplicar algoritmos, en la que aprender un concepto sólo consiste en memorizar su definición lejos de todo contexto, y en donde, saber matemática significa resolver problemas de tipo algorítmico siguiendo las pautas señaladas por el profesor en el aula, sin espacio para la discusión, el error, al análisis y el debate. En tales circunstancias, vale la pena preguntarse: ¿Es necesario un cambio en el ejercicio de la docencia?, ¿Es posible gestionar de otra manera el proceso de enseñanza aprendizaje, brindado a los estudiantes espacios para la discusión y el debate? ¿Es posible que nuestros estudiantes aprendan el concepto de función como producto de su construcción en escenarios contextualizados?.

En virtud a lo anterior se planteó el siguiente problema de investigación: ¿Qué efecto tiene la aplicación de una ingeniería didáctica en el aprendizaje del concepto de función en los estudiantes de la Universidad Nacional Micaela Bastidas de Apurímac?.

El objetivo general fue: Determinar la efectividad de la aplicación de una ingeniería didáctica en el aprendizaje del concepto de función en los estudiantes de la universidad Nacional Micaela Bastidas de Apurímac.

También se plantearon los siguientes objetivos específicos:

Determinar el nivel de aprendizaje de los estudiantes antes de la aplicación de la ingeniería.

Determinar el nivel de aprendizaje de los estudiantes después de la aplicación de la ingeniería.

Determinar el nivel de significancia de la aplicación de la ingeniería didáctica en el aprendizaje del concepto de función.

Evaluar la efectividad de la aplicación de la ingeniería didáctica en relación al aprendizaje del concepto de función.

\section{Ingeniería Didáctica}


Una ingeniería didáctica es un conjunto de secuencias de clase, diseñadas, organizadas y articuladas coherentemente por un "profesoringeniero", para lograr el aprendizaje de cierto conocimiento en un grupo de alumnos específico [1]. Se considera a una ingeniería didáctica como un "producto" que resulta de un análisis preliminar, que considera las dimensiones cognitiva, didáctica y epistemológica del conocimiento a estudiar y luego de un análisis a priori donde se decide sobre las variables didácticas pertinentes. Por otro lado, también se considera como un "proceso" en el cual el docente implementa el producto y realiza los ajustes y adaptaciones necesarias según la dinámica de la clase lo exija.

La Ingeniería Didáctica es un instrumento metodológico para la enseñanza y también para la investigación. Como metodología de investigación, se caracteriza fundamentalmente porque sus productos son construidos a partir de un esquema experimental basado en las realizaciones didácticas en clase, es decir, sobre la concepción, realización, observación y análisis de secuencias de enseñanza; y también porque se ubica en los registros de los estudios de caso y cuya validación es interna, es decir, basada en la confrontación entre el análisis a priori y a posteriori (Artigue, 1995). [1]

La ingeniería Didáctica tiene las siguientes fases: Análisis preliminar: donde se analizan y determinan, desde un enfoque sistémico, todos y cada uno de los actores del sistema didáctico y las relaciones entre los mismo. Implica contemplar la componente epistemológica, cognitiva y didáctica.

Diseño de la situación didáctica y su análisis a priori: donde se eligen las variables didácticas que se controlarán; además, se establecen las hipótesis de trabajo.

Experimentación: donde se pone en escena la situación diseñada en la etapa anterior.

Análisis a posteriori y validación: donde se revisa exhaustivamente los sucesos acaecidos durante la puesta en escena de la situación diseñada. Se confrontan las hipótesis definidas en el análisis a priori y se determina en qué medida las expectativas fueron alcanzadas 0 cuanto se desvían los resultados de lo que se esperaba.

\section{Teoría de las Situaciones Didácticas}

En el modelo de las situaciones Didáctica de G. Brosseau, el proceso de producción de conocimientos matemáticos en una clase se describe a partir de dos tipos de interacciones básicas: La interacción alumno con una problemática que ofrece resistencia y retroacciones que operan sobre los conocimientos matemáticos puestos en juego; y la interacción del docente con el alumno en relación de la interacción del alumno con la problemática matemática propuesta. Según Brosseau, el sujeto produce el conocimiento como resultado de la adaptación a un "medio" resistente con el que interactúa. [2]

En el contexto de la enseñanza, Brosseau distingue en la situación didáctica a los actores (profesor, alumno, medio), una situación inicial, un conjunto de estados posibles (constituyen las estructuras), las reglas que permiten pasar de un estado a otro, las estrategias aplicadas y las decisiones tomadas por el profesor y alumno (constituyen el contexto fenoménico). Brosseau sostiene que en una primera fase para que se dé el aprendizaje, el profesor debe seleccionar problemas donde el alumno debe actuar, reflexionar y evolucionar a su ritmo tratando de adaptarse a la situación y llegar a la solución del mismo. A esta situación la denomina Situación A didáctica, la cual puede ser de tres tipos: de acción (interviene el alumno y el medio), de formulación (interviene el alumno) y de validación (intervienen los alumno y se trata de convencer sobre la validación de la información). En una segunda fase, el profesor intervendrá en la situación estableciendo la institucionalización del conocimiento donde se transformará el conocimiento aprendido en saber científico. [2]

\section{Teoría de los Registros de Representación Semiótica de R. Duval}

Las diferentes experiencias dentro de los salones de clases hacen notar las diversas dificultades que encuentran los estudiantes a la hora de comprender un objeto matemático. Esta comprensión se ve básicamente restringida sólo a la utilización de expresiones algebraicas donde se efectúan ciertos procedimientos algorítmicos. Cuando el estudiante se ve confrontado con otro contexto, se ve limitado en cuanto a su accionar sin lograr avanzar.

Esto lo nota Duval cuando afirma que: “... se ha probado que cambiar la forma de una representación es, para muchos alumnos de los diferentes niveles de enseñanza, una operación difícil y en algunas ocasiones imposible. Todo sucede como si para la gran mayoría de los alumnos la compresión que logran de un contenido quedara limitada a la forma de representación utilizada" [3]. 
Duval plantea la siguiente definición para representaciones semióticas: "Las representaciones semióticas son producciones constituidas por el empleo de signos que pertenecen a un sistema de representación, el cual tiene sus propias restricciones de significado y de funcionamiento.

Una figura geométrica, un enunciado en lengua natural, una fórmula algebraica, una gráfica, son representaciones semióticas que pertenecen a sistemas semióticos diferentes" [4].

Duval sostiene lo siguiente: "Si se llama semiosis a la aprehensión 0 la producción de una representación semiótica, y neosis a la aprehensión conceptual de un objeto, es necesario afirmar que la neosis es inseparable de la semiosis" [3].

En virtud a esto, se concluye que el aprendizaje en matemática pasa por el hecho de distinguir un objeto de su representación, por lo cual, si se le presenta al estudiante una diversidad de registros de representación de un objeto, éste irá consolidando una red conceptual que mejora el nivel de aprendizaje de dicho objeto matemático.

Duval considera que hay dos grandes tipos de transformaciones [4]: El tratamiento: que es una transformación estrictamente interna a un registro; y la conversión que es una transformación de la representación de un objeto en un registro $\mathrm{P}$ es otra representación del mismo objeto en un registro $\mathrm{L}$.

Al respecto Duval plantea lo siguiente: “...la conversión de las representaciones semióticas constituye la actividad cognitiva menos espontánea y más difícil de adquirir para la gran mayoría del estudiante" [4].

Lo anterior evidencia la importancia que tiene para el aprendizaje de las matemáticas que los estudiantes estén en la capacidad de hacer conversiones entre los diferentes registros de representación. Esta conversión es una actividad necesaria para favorecer la actividad conceptual relacionada a cierto contenido matemático.

\section{Metodología}

Se trabajo con una muestra no probabilística conformada por 22 estudiantes de la Escuela Académico Profesional de Ingeniería Agroindustrial pertenecientes al primer semestre de estudios.

Se aplicó un cuestionario para determinar el nivel de aprendizaje de los estudiantes antes de la aplicación de la Ingeniería Didáctica. Este mismo instrumento se aplicó después de la experimentación para determinar los resultados del aprendizaje.

Para determinar la diferenciación del grupo antes y después de la experimentación, se siguió el siguiente procedimiento estadístico:

Para cada alumno se determinó el total de respuestas correctas por indicador. Se calculó la media para respuestas correctas para cada estudiante, antes y después de la aplicación de la ingeniería didáctica. Se aplicó una prueba de hipótesis T de Studens de diferencia de medias, con un nivel de confianza de $95 \%$.

Para determinar la efectividad de la ingeniería didáctica, se procedió de la siguiente manera: Sobre cada indicador se determinó el porcentaje de respuestas correctas del grupo. Se promediaron los porcentajes por cada indicador. Luego, el criterio de decisión fue el siguiente: si el porcentaje es mayor de $70 \%$ la ingeniería didáctica fue efectiva sobre el indicador, en caso contrario no fue efectiva.

\section{Resultados y discusión}

Luego de aplicar la ingeniería didáctica se obtienen los siguientes resultados tanto en el pre test como en el post test.

\section{Resultados del Pre test.}

Tabla 1: Nivel de aprendizaje de los estudiantes obtenidos con la aplicación del pre test

\begin{tabular}{|l|c|c|}
\hline NIVEL DE APRENDIZAJE & $\mathbf{F i}$ & $\mathbf{f i} \%$ \\
\hline MUY BUENO & 0 & 0 \\
\hline BUENO & 1 & 4 \\
\hline REGULAR & 12 & 55 \\
\hline DEFICIENTE & 9 & 41 \\
\hline MUY DEFICIENTE & 0 & 0 \\
\hline TOTAL & $\mathbf{2 2}$ & $\mathbf{1 0 0}$ \\
\hline
\end{tabular}

Se observa que el $96 \%$ de los estudiantes no superan el nivel regular en cuanto al aprendizaje del concepto de función lo cual pone en evidencia las carencias que presentan en cuanto al desarrollo de habilidades para poder trabajar con los diferentes registros de este concepto matemático. Un escaso $4 \%$ muestra un buen nivel de aprendizaje del concepto de función.

\section{Resultados del Post test}


Se observa que el $91 \%$ de los estudiantes han superado el nivel regular en cuanto al aprendizaje Tabla 2: Nivel de aprendizaje de los estudiantes obtenidos con la aplicación del post test

\begin{tabular}{|l|c|c|}
\hline NIVEL DE APRENDIZAJE & $\mathbf{F i}$ & $\mathbf{f i} \%$ \\
\hline MUY BUENO & 2 & 9 \\
\hline BUENO & 18 & 82 \\
\hline REGULAR & 2 & 9 \\
\hline DEFICIENTE & 0 & 0 \\
\hline MUY DEFICIENTE & 0 & 0 \\
\hline TOTAL & $\mathbf{2 2}$ & $\mathbf{1 0 0}$ \\
\hline
\end{tabular}

del concepto de función lo cual muestra que han alcanzado a desarrollar las habilidades necesarias para trabajar con diferentes registros de representación del concepto de función. Sólo dos estudiantes evidencian un nivel regular de aprendizaje, sin embargo, éstos presentaban un nivel deficiente de aprendizaje antes de la aplicación de la ingeniería didáctica.

\section{Análisis para de diferenciación del grupo antes y después de la aplicación de la ingeniería didáctica}

Para este análisis se plantearon las siguientes hipótesis:

Ho: No existen diferencias significativas entre las medias del nivel de aprendizaje antes y después de la aplicación de la ingeniería didáctica.

$\mathrm{Ha}$ : Existen diferencias significativas entre las medias del nivel de aprendizaje antes y después de la aplicación de la ingeniería didáctica. La aplicación de la prueba $\mathrm{T}$ de Students para muestras relacionadas dio el siguiente resultado:

Tabla 3: Resultados de la prueba T de Students para muestras relacionadas

\begin{tabular}{|c|c|c|}
\hline \multicolumn{2}{|l|}{ Media } & -7.864 \\
\hline \multicolumn{2}{|l|}{ Desv. Tipica } & 2.764 \\
\hline \multicolumn{2}{|l|}{ Error Tip media } & 0.59 \\
\hline \multirow{2}{*}{$\begin{array}{l}95 \% \text { intervalo de } \\
\text { confianza }\end{array}$} & Inferior & -9.09 \\
\hline & Superior & -6.638 \\
\hline \multicolumn{2}{|l|}{$\mathrm{T}$} & -13.338 \\
\hline \multicolumn{2}{|l|}{$\mathrm{Gl}$} & 21 \\
\hline \multicolumn{2}{|l|}{ sig(bilateral) } & 0 \\
\hline
\end{tabular}

Como el valor $\mathrm{sig}=0$ es menor que $p=0.005$, se rechaza la hipótesis nula y se acepta la hipótesis alterna. Por lo tanto, existen diferencias significativas entre la media del grupo antes y después de la aplicación de la ingeniería didáctica.

\section{Análisis para la efectividad de la ingeniería didáctica}

Los resultados obtenidos en cuanto a la efectividad de la ingeniería didáctica se muestran en la siguiente figura:

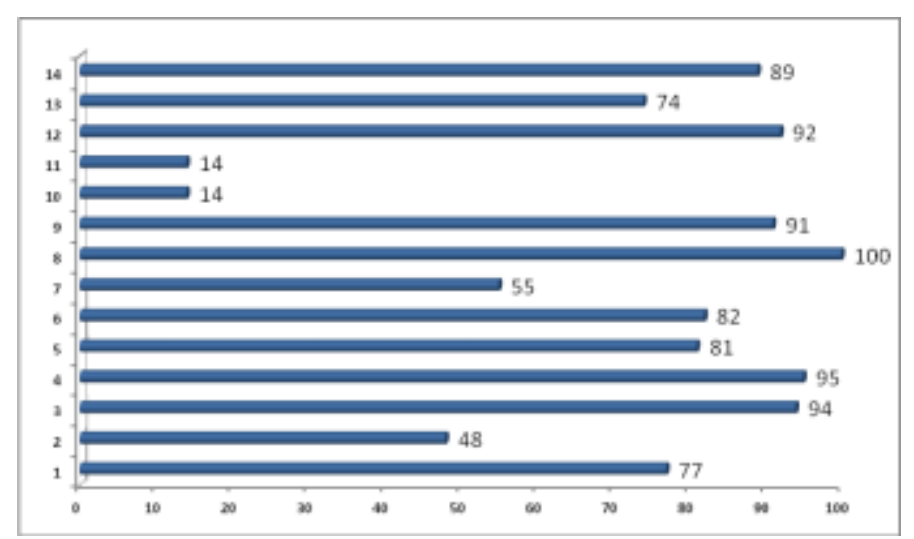

Figura 1: Porcentajes de respuestas correctas por indicador

Los porcentajes de aciertos obtenidos en los indicadores sobre el aprendizaje del concepto de función superan el $70 \%$ en 10 de los 14 indicadores propuestos lo que representa el $71 \%$ del total.

Los indicadores 13 y 14 referidos a la conversión del registro verbal al gráfico y algebraico a través de modelización de situaciones son los que menores porcentajes de aciertos obtuvieron.

En concordancia con lo planteado con Duval, la presentación a los estudiantes de diferentes situaciones que le permita manipular y trabajar con diferentes registros de representación del concepto de función ha permitido una mejora significativa en cuanto al nivel de aprendizaje de los estudiantes en relación a este importante concepto matemático, tal como lo muestran los resultados obtenidos en la tabla 3. La ingeniería didáctica planteada permitió mejorar las habilidades de los estudiantes, que son necesarias para realizar conversiones entre representaciones. El diseño de la ingeniería didáctica tomó como base la clasificación hecha por Janvier, por lo cual, de acuerdo a lo mostrado en la Figura 1, la ingeniería didáctica permitió a los estudiantes mejorar sus habilidades para interpretar gráficas, interpretar fórmulas algebraicas, calcular 
valores, realizar ajustes numéricos lo cual permitió mejorar su aprendizaje sobre el concepto de función. Sin embargo, la habilidad para realizar modelizaciones a partir de un enunciado verbal fue la que menos se desarrolló, lo cual pone en evidencia lo manifestado por Duval cuando señala que las tareas de conversión son menos espontáneas y muy difíciles de adquirir.

\section{Conclusiones}

El nivel de aprendizaje del concepto de función antes de la aplicación de la ingeniería didáctica no supera el nivel regular en casi todos los estudiantes.

El nivel de aprendizaje del concepto de función después de la aplicación de la ingeniería didáctica es superior al nivel regular en casi todos los estudiantes que participaron en la experiencia.

La aplicación de la ingeniería didáctica basada diseñada en los registros de representación del concepto de función mejoró significativamente el aprendizaje de este concepto matemático en los estudiantes de ingeniería agroindustrial del I ciclo de la Universidad Nacional Micaela Bastidas.

La aplicación de la ingeniería didáctica fue efectiva pues permitió mejorar habilidades importantes que le permiten al estudiante realizar conversiones entre los diferentes registros de representación del concepto de función.

La comprensión del concepto de función en los estudiantes se ha visto fortalecida por la manipulación de los diferentes tipos de representación de este concepto

\section{Agradecimientos}

A los estudiantes de la Escuela Académico Profesional de Ingeniería Agroindustrial de la Universidad Nacional Micaela Bastidas de Apurímac por su participación en este trabajo.

\section{Referencias}

[1] M. Artigue y A. Douady, Ingeniería Didáctica en Educación Matemática, Edit. Iberoamericana. México, 1995

[2] G. Brosseau, Fundamentos y Métodos de la Didáctica de la Matemática. Facultad de Matemática, Astronomía y Física. Córdoba, 1986.

[3] R. Duval, Semiosis y Pensamiento Humano. Registros semióticos y Aprendizaje Intelectual, Universidad del Valle. Colombia. 2004

[4] R. Duval, Los problemas Fundamentales en el Aprendizaje de las Matemáticas y las Formas Superiores del Desarrollo Cognitivo, Universidad del Valle. Colombia. 1999

[5] R. Duval, Registros de Representación Semiótica y Funcionamiento Cognitivo del Pensamiento, Didáctica, Investigaciones en Matemática Educativa, Grupo Editorial Iberoamérica, S.A. de C.V., México. 1999

E-mail: pex 2008@hotmail.es 